\title{
Editorial: In-situ and In-operando Techniques for Material Characterizations During Battery Operation
}

\author{
Verónica Palomares ${ }^{1,2 \star}$ and Neeraj Sharma ${ }^{3}$ \\ ${ }^{1}$ Inorganic Chemistry Department, University of the Basque Country/Euskal Herriko Unibertsitatea UPV/EHU, Bilbao, Spain, \\ ${ }^{2}$ BCMaterials, BId. Martina Casiano, UPV/EHU Science Park, Leioa, Spain, ${ }^{3}$ School of Chemistry, University of New South \\ Wales, Sydney, NSW, Australia
}

Keywords: operando and in situ measurements, battery, materials characterization techniques, electrochemistry, structure-property relationship

Editorial on the Research Topic

In-situ and In-operando Techniques for Material Characterizations During Battery Operation

OPEN ACCESS

Edited by:

Soorathep Kheawhom,

Chulalongkorn University, Thailand

Reviewed by:

Ryan Corpuz,

Mindanao State University, lligan Institute of Technology, Philippines

Maiyalagan Thandavarayan,

University of Texas at Austin,

United States

*Correspondence:

Verónica Palomares

veronica.palomares@ehu.eus

Specialty section:

This article was submitted to Electrochemical Energy Conversion and Storage,

a section of the journal Frontiers in Energy Research

Received: 14 January 2019

Accepted: 28 January 2019

Published: 15 February 2019

Citation:

Palomares V and Sharma N (2019)

Editorial: In-situ and In-operando Techniques for Material

Characterizations During Battery

Operation. Front. Energy Res. 7:10.

doi: 10.3389/fenrg.2019.00010
Batteries are prevalent in society and there is a need to develop better batteries to meet future demands. To build better batteries it is crucial to understand how components within real devices function and interact during use. This provides significantly more insight into function compared to isolated and idealized laboratory conditions. Electrochemical cells, whether half cells or full cells can be developed at various scales, depending on the desired application or research question. Electrochemical methods provide insight on the reactions and the potentials at which they happen, energy storage density, retention over cycling, rate capability to name a few parameters. They can be used to derive the impedance of the cell and back calculated to investigate interfacial and charge resistance phenomena. These methods are critical in understanding the electrochemistry of the batteries and are being widely used in research and development, however they only provide a narrow view of the materials response and function in the device. If the electrochemistry can be coupled with another analytical technique, such as spectroscopy, imaging, or diffraction while the device is operating, the insight gained into materials function is enhanced. The additional analytical techniques can be used to understand the electrochemistry with a totally different perspective, for example an atomic scale perspective on electrochemistry.

It is this ability to gain unparalleled insight into materials function within devices that have led researchers to push the boundaries of in situ and operando methods. These methods couple the electrochemical measurements or stimuli with another analytical technique(s). They often gain both electrochemical and structural or spectroscopic data which can be directly correlated. Thus providing two or more views on the function of the material and increasing the parameter space that can be measured in one experiment and correlated. These experiments by definition need to be conducted in an electrochemical cell environment (to run electrochemistry) while another measurement is occurring, with say X-rays or laser light impinging on the electrode of interest to through the entire device. Ideally full cells or commercially equivalent batteries should be used, however these in most cases are perfected for electrochemistry but not very well suited for other analytical methods. Therefore, specially designed electrochemical cells are often used, where the cell is optimized to achieve the best from both worlds, electrochemistry and the other analytical technique. This typically requires compromises, reducing the electrochemical performance to obtain better signal from the analytical method or vice versa. However, researchers are constantly trying to minimize the changes and maximize both the electrochemical performance and signal observed in the analytical method, 
thereby obtaining the best of both worlds. It is interesting to note, how routine such in situ and operando measurements are becoming for techniques like in situ X-ray diffraction, Raman spectroscopy, or FTIR as examples. With such works catalyzing significant development in relatively newer methods such as in situ electron microscopy.

The field is truly very exciting, new developments, new methods, combining of techniques, and gaining amazing insight into materials function in devices.

This selection of articles explores a variety of in situ characterization techniques, such as operando X-ray or neutron powder diffraction, Raman spectroscopy, neutron depth profiling, or in situ transmission X-ray microscopy. These techniques are analyzed from different approaches, either as original research work, review, or perspective article and cover very different battery systems, from more classical Li-ion half and full cell batteries, all solid state thin film batteries, or high temperature thermal batteries to newer systems such as air cathode batteries.
We hope that you enjoy these articles and appreciate the great work these researchers and other researchers engaged in in situ and operando methods are doing both from method development to a materials function point of view. We also thank the authors and reviewers for their contributions to this work.

\section{AUTHOR CONTRIBUTIONS}

All authors listed have made a substantial, direct and intellectual contribution to the work, and approved it for publication.

Conflict of Interest Statement: The authors declare that the research was conducted in the absence of any commercial or financial relationships that could be construed as a potential conflict of interest.

Copyright (c) 2019 Palomares and Sharma. This is an open-access article distributed under the terms of the Creative Commons Attribution License (CC BY). The use, distribution or reproduction in other forums is permitted, provided the original author(s) and the copyright owner(s) are credited and that the original publication in this journal is cited, in accordance with accepted academic practice. No use, distribution or reproduction is permitted which does not comply with these terms. 